\title{
Theoretical stability, thin film synthesis and transport properties of the Mon+1GaCn MAX phase
}

\author{
Rahele Meshkian, Arni Sigurdur Ingason, Martin Dahlqvist, Andrejs Petruhins, Unnar B. \\ Arnalds, Fridrik Magnus, Jun Lu and Johanna Rosén
}

\section{Linköping University Post Print}

\section{Tweet}

N.B.: When citing this work, cite the original article.

Original Publication:

Rahele Meshkian, Arni Sigurdur Ingason, Martin Dahlqvist, Andrejs Petruhins, Unnar B. Arnalds, Fridrik Magnus, Jun Lu and Johanna Rosén, Theoretical stability, thin film synthesis and transport properties of the Mon $+1 \mathrm{GaCn}$ MAX phase, 2015, Physica Status Solidi. Rapid Research Letters, (9), 3, 197-201. http://dx.doi.org/10.1002/pssr.201409543

Copyright: Wiley-VCH Verlag http://www.wiley-vch.de/publish/en/

Postprint available at: Linköping University Electronic Press http://urn.kb.se/resolve?urn=urn:nbn:se:liu:diva-117388 


\title{
Theoretical stability, thin film synthesis and transport properties of the $\mathrm{Mo}_{n+1} \mathrm{GaC}_{n}$ MAX phase
}

\author{
Rahele Meshkian*,1, Arni Sigurdur Ingason", Martin Dahlqvist ${ }^{1}$, Andrejs Petruhins ${ }^{1}$, Unnar B. Arnalds ${ }^{2}$, \\ Fridrik Magnus ${ }^{3}$, Jun Lu', Johanna Rosen ${ }^{1}$ \\ ${ }^{1}$ Department of Physics, Chemistry and Biology (IFM), Linköping University, SE-581 83 Linköping, Sweden \\ ${ }^{2}$ Science Institute, University of Iceland, IS-107 Reykjavik, Iceland \\ ${ }^{3}$ Department of Physics and Astronomy, Uppsala University, Box 516, SE-516 Uppsala, Sweden
}

Keywords Superconducting MAX phase, ab-initio calculation, magnetron sputtering, thin film synthesis

*Corresponding author: e-mail rahme@ifm.liu.se

The phase stability of $\mathrm{Mo}_{n+1} \mathrm{GaC}_{n}$ has been investigated using ab-initio calculations. The results indicate stability for the $\mathrm{Mo}_{2} \mathrm{GaC}$ phase only, with a formation enthalpy of $-0.4 \mathrm{meV}$ per atom. Subsequent thin film synthesis of $\mathrm{Mo}_{2} \mathrm{GaC}_{\mathrm{Cas}}$ performed through magnetron sputtering from elemental targets onto $\mathrm{Al}_{2} \mathrm{O}_{3}$ [0001], $6 \mathrm{H}-\mathrm{SiC}$ [0001] and $\mathrm{MgO}$ [111] substrates within the temperature range of 500 and $750^{\circ} \mathrm{C}$. High structural quality films were obtained for synthesis on $\mathrm{MgO}$ [111] substrates at $590{ }^{\circ} \mathrm{C}$. Evaluation of transport properties showed a superconducting behavior with a critical temperature of approximately $7 \mathrm{~K}$, reducing upon the application of an external magnetic field. The results point towards the first superconducting MAX phase in thin film form.

$M_{n+1} A X_{n}$ (n=1-3) phases constitute a family of atomically laminated materials based on an early transition metal $(M)$, an A-group element $(A)$ and carbon or/and nitrogen $(X)$. These compounds crystalize in a hexagonal structure belonging to the space group P6 $/ \mathrm{mmc}$ (194) with the $A$ layer interleaved between layers of $M_{n+1} X_{n}$. The laminated structure leads to a combination of metallic and covalent binding between the individual elements, resulting in a combination of both ceramic and metallic properties. For instance, selected MAX phases display high thermal and electrical conductivity, oxidation resistance and stiffness, while being easily machinable [1-3]. MAX phases, at the time denoted H-phases $\left(\mathrm{M}_{n+1} \mathrm{AX} n, n=1\right)$, were discovered in the 1960's by Nowotny et al. [4] and more than thirty phases were synthesized. Interest was reignited a few decades later by the work of Barsoum et al. [5], leading to the nomenclature $M_{n+1} A X_{n}$ phases.

Predicting and synthesizing unexplored MAX phases is motivated by the number of possible isostructural compositions, ensuring a wide range of different properties. $\mathrm{Mo}_{2} \mathrm{GaC}$ is a phase where theory suggests superconducting behavior [6]. Comparing with $\mathrm{Nb}_{2} \mathrm{GaC}$ and $\mathrm{V}_{2} \mathrm{GaC}$, also theoretically predicted to be superconducting, the band structure suggest a higher degree of anisotropy for $\mathrm{Mo}_{2} \mathrm{GaC}$, similar to e.g. $\mathrm{Ti}_{2} \mathrm{Al}\left(\mathrm{C}_{\mathrm{x}} \mathrm{O}_{1-}\right.$ x) [7]. Several bulk MAX phases show superconductivity, namely $\mathrm{Nb}_{2} \mathrm{SC}$ [8], $\mathrm{Nb}_{2} \mathrm{SnC}$ [9], $\mathrm{Nb}_{2} \mathrm{AsC}$ [10], $\mathrm{Ti}_{2} \operatorname{InC}$ [11], $\mathrm{Nb}_{2} \operatorname{InC}$ [12] and $\mathrm{Ti}_{2} \mathrm{InN}$ [13]. However, the result found by Bortolozo et al. [12, 13] has to date not been reproduced. In addition, Barsoum [14] have reported that $\mathrm{Nb}_{2} \mathrm{SnC}$ and $\mathrm{Ti}_{2} \mathrm{InC}$ do not display any superconducting behaviour. Furthermore, there is to date no superconducting MAX phase in thin film form.

Synthesis of bulk $\mathrm{Mo}_{2} \mathrm{GaC}$ has previously been carried out by L. E. Toth [15] in 1967, where an evacuated quartz capsule with powders of molybdenum and carbon and with liquid gallium was heated at $850{ }^{\circ} \mathrm{C}$ for a duration of four weeks. The transport measurements performed on the sample showed a superconducting critical temperature at $3.9 \mathrm{~K}$ [15]. However, no phase and composition analysis, such as X-ray diffraction (XRD) or transmission electron microscopy (TEM) is performed, making it difficult to draw any conclusions regarding the purity or structural quality of the synthesized material. The presence of superconducting $\mathrm{MoC}$ as well as $\mathrm{Mo}_{2} \mathrm{C}$ may influence the results, as these phases crystallize in various different structures, with measured critical temperatures from 6 up to $13 \mathrm{~K}[16,17]$. Thus, careful evaluation of the transport properties ideally requires a highly oriented single crystal sample with minimized defect concentrations and grain boundaries in order to avoid any result that might stem from possible impurity carbides in addition to the MAX phase. There are no reports on thin film synthesis of $\mathrm{Mo}_{2} \mathrm{GaC}$, which makes it an excellent candidate for a detailed investigation on synthesis procedures, materials optimization, and transport properties.

We have in the present study performed theoretical evaluation of the phase stability of $\mathrm{Mo}_{n+1} \mathrm{GaC}_{n}$, with subsequent thin film synthesis of $\mathrm{Mo}_{2} \mathrm{GaC}$. The theoretical work also identified the most competing phases, and their stability, to be used as guidance in the material synthesis and analysis.

Evaluation of transport properties shows a superconducting response with a transition temperature at $7 \mathrm{~K}$, displaying a clear reduction in $T_{\mathrm{c}}$ upon the application of an external magnetic field in the range 0 to $5 \mathrm{~T}$. 
DC magnetron sputtering was used for thin film synthesis, involving three elemental targets, molybdenum (Mo), gallium (Ga) and carbon (C). Due to Ga being liquid close to room temperature (melting point $30{ }^{\circ} \mathrm{C}$ ), previously developed procedures for MAX phase synthesis involving liquid targets were utilized [18]. The base pressure was $6 \cdot 10^{-8}$ Torr and during deposition the Ar-gas pressure was 4.8 mTorr. The net flux of the target material was calibrated by performing room temperature depositions for Mo and $\mathrm{C}$ targets at different current/power. Density and thickness of the films were obtained by X-ray reflectivity (XRR). Further optimization of the deposition procedure was done by XRD on deposited test samples. Depositions of $\mathrm{Mo}_{2} \mathrm{GaC}_{\mathrm{C}}$ were performed on $\mathrm{Al}_{2} \mathrm{O}_{3}$ [0001], 6H-SiC [0001] and $\mathrm{MgO}$ [111] substrates. These were cleaned in an ultrasonic bath in acetone, ethanol and isopropanol for duration of ten minutes each. The substrates were afterwards annealed at the growth temperature of the MAX phase $\left(590^{\circ} \mathrm{C}\right)$ inside the vacuum chamber for 15 minutes prior to deposition. Structural characterization of the optimized thin films was obtained by XRD. For that purpose a Panalytical Empyrean MRD with a line focus $\mathrm{Cu} \mathrm{K} \mathrm{K}_{\alpha}$ source $(\lambda=1.5418 \AA$ ) was employed. For the symmetric $(\theta-2 \theta)$ measurements the system was equipped with a hybrid mirror and a $0.27^{\circ}$ parallel plate collimator in the incident and diffracted beam side, respectively. For pole measurements, the source was set to the point focus position with a capillary lens used in the incident beam side. Detailed structural analysis was carried out using TEM. Preparation of a cross-sectional TEM-sample was accomplished using conventional mechanical methods with additional low angle ion milling, finalized by a low acceleration voltage fine-polishing step. The TEM used for this study was FEI Tecnai G2 TF20 UT instrument with a field-emission gun, operated at $200 \mathrm{kV}$, including energy dispersive spectroscopy (EDX) and point resolution of $0.19 \mathrm{~nm}$. A four point probe was used for evaluating the resistivity of the film as a function of temperature from room temperature down to $3 \mathrm{~K}$, and in an externally applied magnetic field up to $5 \mathrm{~T}$, parallel to the plane of the film.

All calculations performed were based on Density Functional Theory (DFT) and the projector augmented wave (PAW) method [19] as implemented in the Vienna ab initio simulation package, VASP [20, 21]. The exchangecorrelation energy and electron potential were accounted for by employing the generalized gradient approximation (GGA) and the Perdew-Burke-Ernzerhof exchange (PBE) model [22]. The Monkhorst pack scheme [23] was utilized for integrating reciprocal space. The plane wave cutoff energy was set to $400 \mathrm{eV}$ with a convergence of about $0.1 \mathrm{meV}$ for the total energy. The same convergence was used for the $k$-point density.

The formation enthalpy of $\mathrm{Mo}_{n+1} \mathrm{GaC}_{n}$ was evaluated according to previously developed procedures [7, 24, 25]. All experimentally observed competing phases within the Mo-Ga-C system were identified from phase diagrams and databases. Also a set of hypothetical phases were included in the calculations, based on observations in neighboring materials systems. All phases included in the present study are listed in Table S1, (see Supplemental Material). A linear optimization procedure based on the simplex method was used for identifying the set of most competing phases under the constraints of a MAX phase stoichiometry. The formation enthalpy for the MAX phase, $\Delta H_{M_{n+1} A X_{n}}$, can then be formulated as,

$$
\Delta H_{M_{n+1} A X_{n}}=\left(E_{M_{n+1} A X_{n}}-\sum E_{c p}\right) / 2(n+1)
$$

where $E_{M_{n+1} A X_{n}}$ is the energy of the $M_{n+1} A X_{n}$ phase and the sum of $E_{\mathrm{cp}}$ is the energy for the set of competing phases. By dividing the expression with $2(n+1)$ the formation energy per atom is obtained.

Including all known phases in the Mo-Ga-C system, see Table S1, (see Supplemental Material), $\mathrm{Mo}_{2} \mathrm{GaC}_{\text {is }}$ predicted stable compared to the set of most competing phases $\mathrm{Mo}_{3} \mathrm{Ga}, \mathrm{Mo}_{6} \mathrm{Ga}_{31}$, and $\gamma-\mathrm{MoC}$, with a formation enthalpy of $-0.4 \mathrm{meV} /$ atom. However, by including hypothetical phases, such as $\mathrm{MoGa}_{4}$ suggested from the cubic $\mathrm{CrGa}_{4}$ observed in the $\mathrm{Cr}-\mathrm{Ga}$ phase diagram, the formation enthalpy of the 211 phase becomes positive, $\sim 3.4 \mathrm{meV} /$ atom, with a new set of most competing phases, $\mathrm{Mo}_{3} \mathrm{Ga}, \gamma-\mathrm{MoC}$ and $\mathrm{MoGa}_{4}$. In order to investigate how sensitive the formation enthalpy is with respect to the identified most competing phases, each phase was excluded and a new set was identified from the remaining phases. However, no other set of competing phases altered the stability result. $\mathrm{Mo}_{3} \mathrm{GaC}_{2}$ and $\mathrm{Mo}_{4} \mathrm{GaC}_{3}$ were predicted unstable, with a formation energy of 102 and $138 \mathrm{meV} /$ atom, respectively, with respect to the same set of most competing phases, $\gamma-\mathrm{MoC}$ and $\mathrm{Mo}_{2} \mathrm{GaC}_{\text {. }}$

Figure 1 shows $\theta$-2 $\theta$ diffractograms from XRD measurements on films deposited on (a) $\mathrm{MgO}$ and (b) $\mathrm{Al}_{2} \mathrm{O}_{3}$ substrates simultaneously. The inset in Fig. 1(a) is a pole figure of the (0006) plane suggesting the formation of tilted grains in the film. For the film deposited on $\mathrm{MgO}$, the basal plane peaks $(000 l)$ of $\mathrm{Mo}_{2} \mathrm{GaC}$ are clearly visible, indicating the preferred orientation of the film. The two additional peaks situated at $36.7^{\circ}$ and $78.4^{\circ}$ are contributions from the (111) and (222) planes of the substrate. Also noticeable is the broadening of the obtained peaks which could possibly be due to the overlap between the planes of the observed tilted grains and the basal planes of the crystal. The measured in-plane and out-of-plane lattice parameters are $a=2.97$ and $c=13.43 \AA$, 
respectively, which is below and above the calculated values of 3.07 and $13.27 \AA$, respectively. Due to the close in-plane parameter of the MAX phase to the (110) spacing of the MgO substrate, $a$, was obtained from indirect measurements of the (1013) plane parameters.

Highest structural quality was obtained at a deposition temperature of about $590^{\circ} \mathrm{C}$. Nevertheless, minor traces of one of the most competing phases $\mathrm{Mo}_{3} \mathrm{Ga}$ can be identified to the left of the substrate peaks in the XRD scan, see Fig. 1(a), a phase which grow in intensity upon both an increase and a reduction in deposition temperature.

Synthesis within a temperature range of 500 and $750{ }^{\circ} \mathrm{C}$ show that the formation of $\mathrm{Mo}_{2} \mathrm{GaC}$ is very sensitive to the growth temperature, and limited to within a narrow window between 580 and $620^{\circ} \mathrm{C}$. Depositions at higher or lower temperatures show strongly reduced MAX phase intensity, indicating a low stability of $\mathrm{Mo}_{2} \mathrm{GaC}$ with respect to its most competing phases, which is consistent with the calculated small formation enthalpy.

Films deposited on $\mathrm{Al}_{2} \mathrm{O}_{3}$ substrates, see Fig. 1(b), showed drastically reduced MAX phase content, with formation of additional intermetallic and carbide phases. Furthermore, for $6 \mathrm{H}-\mathrm{SiC}$ [0001] substrates with an $a$ parameter of $\sim 3.07 \AA$, the obtained result was close to that shown in (b), i.e. of very low MAX phase quality.

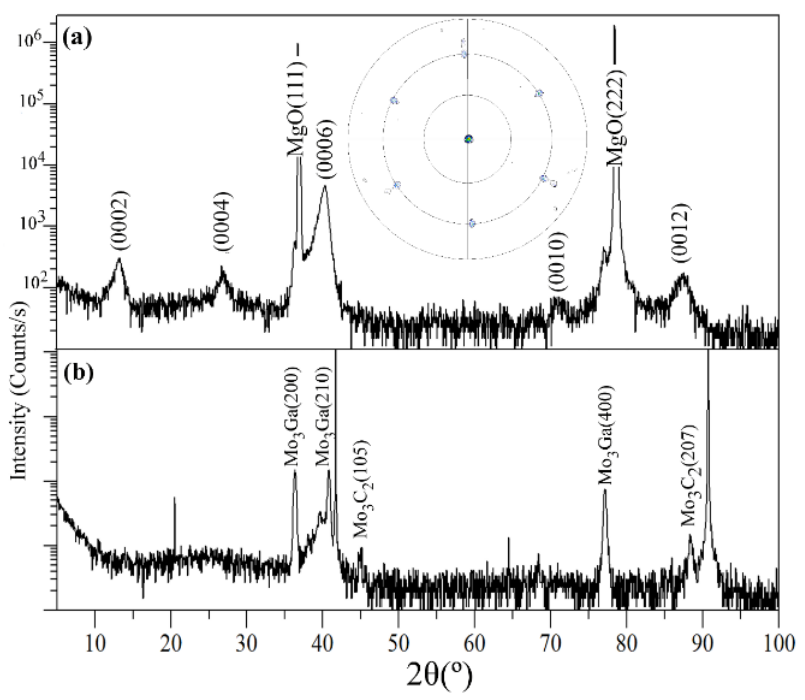

Figure $12 \theta \mathrm{XRD}$ scan from a Mo2 $\mathrm{GaC}$ thin film deposited on (a) $\mathrm{MgO}$ (111) (b) $\mathrm{Al} 2 \mathrm{O} 3$ (0001) for 30 minutes. The inset in (a) shows the pole measurement of the (0006) plane, $2 \theta$ at $40.256^{\circ}$, revealing the presence of tilted grains in the film.

Figure 2 shows TEM micrographs of a film deposited on MgO. In (a) an overview image of the film (top) and the substrate (bottom) can be seen. The image reveals an epitaxially grown film consisting of homogenous crystal structure up to approximately $15 \mathrm{~nm}$, followed by formation of tilted grains. In the higher magnification TEM image, Fig. 2(b), the atomic laminated nature of the MAX phase is apparent. This data confirms the presence of the tilted grains and defects in the film, consistent with the peak broadening seen in the XRD scan. Figure 2(c) shows the associated FFT image obtained from (b). There were no traces of $\mathrm{Mo}_{3} \mathrm{Ga}$ phase in the obtained TEM images. Additional information regarding local composition of the MAX phase was obtained from TEM/EDX, where the ratio of Mo and Ga was determined to 65:35, hence close to a 2:1 ratio, as expected. The total thickness of the film was estimated to be $90 \mathrm{~nm}$ for 30 minutes of deposition, resulting in growth rate of $\sim 0.5 \AA$ per second. 


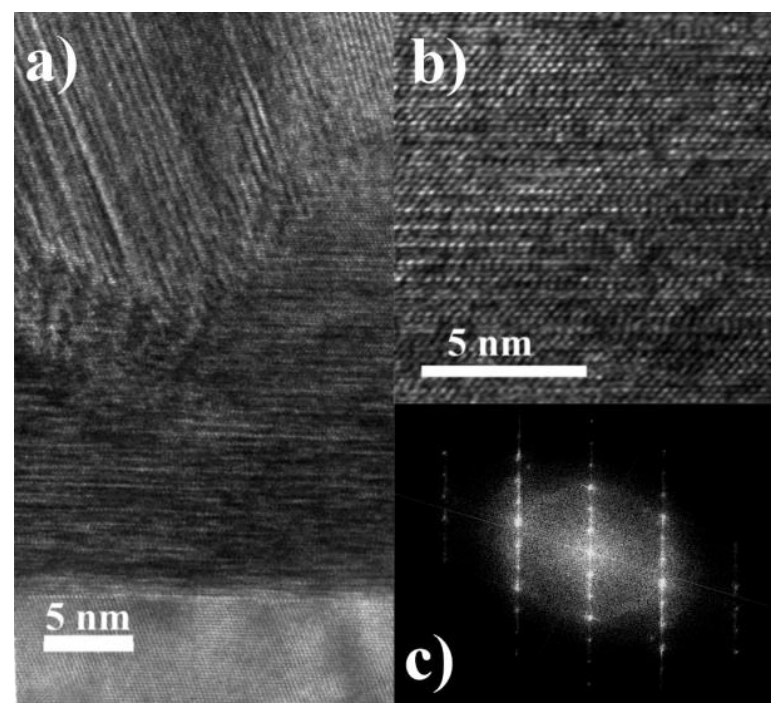

Figure $2 \mathrm{TEM}$ analysis on a $\mathrm{Mo}_{2} \mathrm{GaC}$ film grown for $30 \mathrm{~min}$. a) Low magnification image with the substrate (bottom) and the film (top) revealing the epitaxial growth of the film up to about $15 \mathrm{~nm}$ thickness, followed by the formation of tilted grains. b) Higher magnification micrograph of the epitaxially grown layers showing the laminated nature of the MAX phase with c) associated ED imaging showing the diffraction of the crystal planes.

The temperature dependence of the electrical resistivity of a $90 \mathrm{~nm}$ thick film is shown in Fig. 3(a). The film displays a metallic behavior from $300 \mathrm{~K}$ down to $7 \mathrm{~K}$ where an abrupt drop is observed in the resistivity indicative of a superconducting transition. For the resistivity measurements shown, a current of $100 \mu \mathrm{A}$ was applied through the film. The identified transition temperature was not affected by the applied current in the range $10 \mu \mathrm{A}$ to 10 $\mathrm{mA}$. The width of the transition is over $3 \mathrm{~K}$ and it does not appear to be complete at the lowest measured temperature of $3 \mathrm{~K}$. Upon the application of an external magnetic field, parallel to the film plane, the transition displays a systematic shift to lower temperatures as shown in Fig. 3(b). This behavior is consistent with that of a superconducting transition. The inset of Fig. 3(b) shows the $H-T$ phase diagram of the superconductor from which we can estimate that the zero temperature critical field $H_{c 2}$ is of the order of $10 \mathrm{~T}$ [26]. The previously reported transition temperature of bulk $\mathrm{Mo}_{2} \mathrm{GaC}$ is about $3.9 \mathrm{~K}$ [15], which is considerably lower than the reported value here of $7 \mathrm{~K}$. However, as previously mentioned, there is no structural or compositional analysis allowing a comparison between this material and the thin films of the present paper. It is well known that superconductivity is highly sensitive to composition and crystal structure of a phase [27], and that critical transitions are dependent on the film thickness and its crystal orientation [28]. This can explain the difference in the observed transition temperature. In fact, our detailed XRD and TEM analysis does not show any evidence of other known superconducting phases in our samples, suggesting that the observed transition can be attributed to the MAX phase. The large width of the superconducting transition, as well as the fact that the resistance drop is rather small, is consistent with the two-dimensional character of the MAX phase laminae $[29,30]$. In any case, it is clear that further investigations are required to determine the precise nature of the superconductivity in this complex material. 

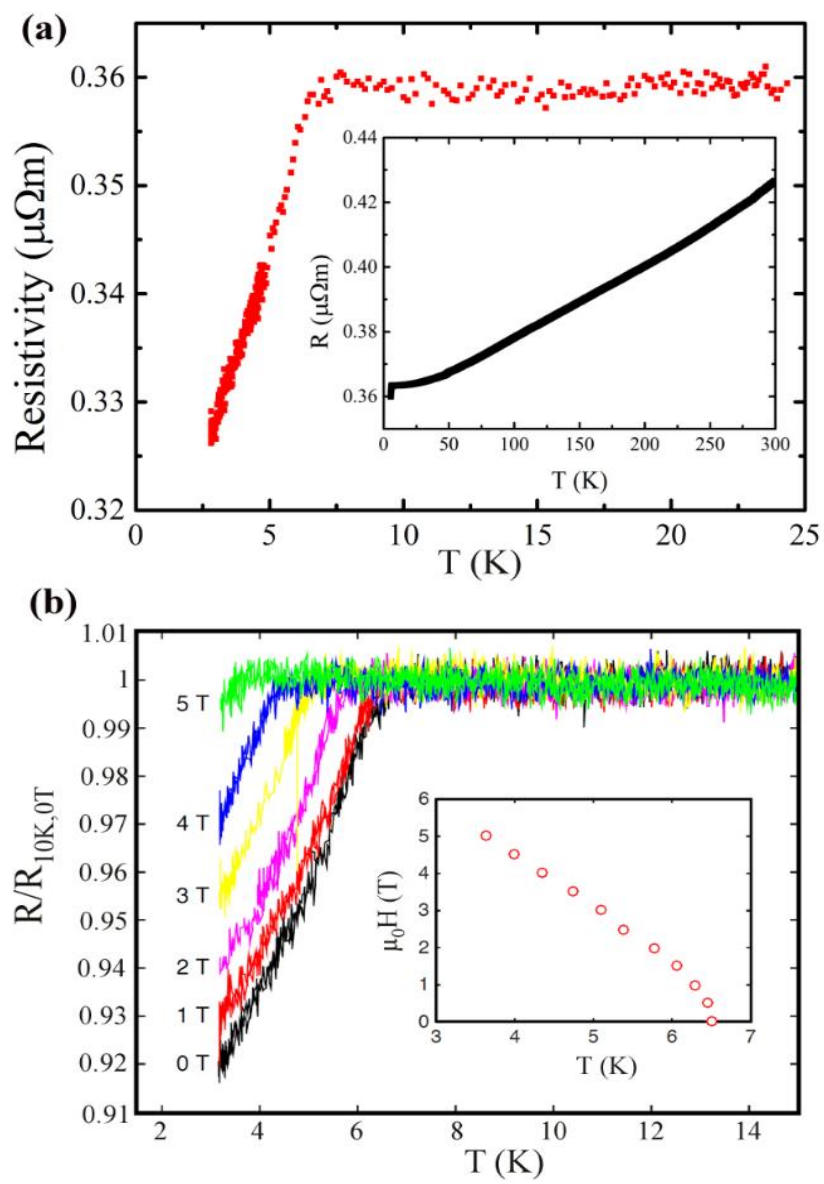

Figure 3 (a) Low temperature dependence of the resistivity for a $90 \mathrm{~nm}$ thick $\mathrm{Mo}_{2} \mathrm{GaC}$ film. At elevated temperature the sample displays metallic behavior, and a transition to a superconducting state at approximately $7 \mathrm{~K}$. (b) Magnetic field dependence of the normalized resistivity and the critical temperature of the film. The magnetic field is parallel to the film plane. The inset shows the $H-T$ phase diagram of the superconductor.

In conclusion, we have investigated the theoretical phase stability of $\mathrm{Mo}_{n+1} \mathrm{GaC}_{n}$, and found $\mathrm{Mo}_{2} \mathrm{GaC}_{\text {to }}$ be stable compared to all known competing phases in the Mo-Ga-C system. Subsequent thin film synthesis through magnetron sputtering reveal epitaxial growth of $\mathrm{Mo}_{2} \mathrm{GaC}$ MAX phase on $\mathrm{MgO}$ [111] substrates. Evaluation of the transport properties shows superconducting behavior with a critical temperature around $7 \mathrm{~K}$, which points towards the first superconducting MAX phase in thin film form.

The research was funded by the European Research Council under the European Community Seventh Framework Program (FP7/2007-2013)/ERC Grant agreement no [258509]. J. Lu acknowledges the KAW Foundation for the Ultra Electron Microscopy Laboratory in Linköping. J. Rosen acknowledges funding from the Swedish Research Council (VR) grant no. 642-2013-8020 and 621-2012-4425, from the KAW Fellowship program, and from the SSF synergy grant FUNCASE. U. B. Arnalds acknowledges funding from the Icelandic Research Fund.

\section{References}

[1] M. W. Barsoum, et al., Annu. Rev. Mater. Res. 41, 195 (2011).

[2] M. Radovic, et al., Am. Ceram. Sci. Bull. 92, 20 (2013).

[3] M.W. Barsoum, et al., Am. Sci. 89, 334 (2001).

[4] W. Jeitschko, et al., Monatsh Chem. 94, 844 (1963).

[5] M.W. Barsoum, Prog. Solid st. Chem. 28, 201 (2000).

[6] I.R. Shein, et al., Phys. C. 470, 533 (2010).

[7] J. Rosen, et al., Appl. Phys. Lett. 97, 073103 (2010).

[8] K. Sakamaki, et al., Solid State Commun. 112, 323 (1999).

[9] A.D. Bortolozo, et al., Solid State Commun. 139, 57 (2006).

[10] S. Lofland, et al., Phys. Rev. B 74, 174501 (2006).

[11] A.D. Bortolozo, et al., Solid State Commun. 144, 419 (2007).

[12] A.D. Bortolozo, et al., Phys. C. 469, 256 (2009). 
[13] A.D. Bortolozo, et al., Solid State Commun. 150, 1364 (2010).

[14] M.W. Barsoum, MAX Phases: Properties of Machinable Ternary Carbides and Nitrides, (Wiley-VCH, Weinheim, 2013), p. 181.

[15] L.E. Toth, J. Less-Common Met. 13, 127 (1967).

[16] L. E. Toth, et al., J. Phys. Chem. Solids 26, 517 (1965).

[17] N. Morton, et al., J. Less-Common Met. 25, 97 (1971).

[18] A. Petruhins, et al., Phys. Status Solidi RRL 7, 971 (2013).

[19] P.E.Blöchl, Phys. Rev. B 50, 17953 (1994).

[20] G. Kresse, et al., Phys. Rev. B 48, 13115 (1993).

[21] G. Kresse, et al., Phys. Rev. B 49, 14251 (1994).

[22] J.P. Perdew, et al., Phys. Rev. Lett. 77, 3865 (1996).

[23] H.J. Monkhorst, et al., Phys. Rev. B 13, 5188 (1976).

[24] M. Dahlqvist, et al., Phys. Rev. B 81, 024111 (2010).

[25] M. Dahlqvist, et al., Phys. Rev. B 81, 220102 (2010).

[26] N.R. Werthamer, et al., Physical Review 147, 288 (1966).

[27] Y. Yosida, et al., Phys. C. 442, 97 (2006).

[28] M.J. Wang, et al., Phys. Rev. Lett. 103, 117002 (2009).

[29] C.G. Granqvist, et al., Phys. cond. Matt 18, 79 (1974).

[30] S. Lee, et al., Phys. Rev. Lett. 64, 3078 (1990). 


\section{Supplemental Material}

\section{Theoretical stability, thin film synthesis and transport properties of the $\mathrm{Mo}_{n+1} \mathrm{GaC}_{n}$ MAX phase}

Rahele Meshkian*,1, Arni Sigurdur Ingason ${ }^{1}$, Martin Dahlqvist ${ }^{1}$, Andrejs Petruhins ${ }^{1}$, Unnar B. Arnalds ${ }^{2}$, Fridrik Magnus ${ }^{3}$, Jun Lu' ${ }^{1}$, Johanna Rosen ${ }^{1}$

${ }^{1}$ Department of Physics, Chemistry and Biology (IFM), Linköping University, SE-581 83 Linköping, Sweden

${ }^{2}$ Science Institute, University of Iceland, IS-107 Reykjavik, Iceland

${ }^{3}$ Department of Physics and Astronomy, Uppsala University, Box 516, SE-516 Uppsala, Sweden

Table S1 List of all competing phases (in addition to the $\mathrm{M}_{n+1} \mathrm{AX}_{n}$ phases) included in the evaluation of phase stability. Phases in italic are hypothetical, i.e. not reported experimentally.

\begin{tabular}{|c|c|c|}
\hline Phase & $\begin{array}{l}\text { Prototype } \\
\text { structure }\end{array}$ & Space group \\
\hline Mo & $\mathrm{W}$ & Im-3m (229) \\
\hline $\mathrm{a}-\mathrm{Ga}$ & $\mathrm{Ga}$ & Cmca (64) \\
\hline $\mathrm{C}$ & $\mathrm{C}$ (graphite) & $\mathrm{P6}_{3} / \mathrm{mmc}(194)$ \\
\hline $\mathrm{Mo}_{3} \mathrm{GaC}$ & CaTiO3 & $P m-3 m(221)$ \\
\hline $\mathrm{MoC}$ & TiP & $\mathrm{P6}_{3} / m m c(194)$ \\
\hline $\mathrm{MoC}$ & $\gamma^{\prime}-\mathrm{MoC}$ & $\mathrm{P6}_{3} / m m c(194)$ \\
\hline $\mathrm{MoC}$ & $\mathrm{NaCl}$ & $F m-3 m(225)$ \\
\hline $\mathrm{MoC}$ & $\eta-\mathrm{MoC}$ & $P 6_{3} / m m c(194)$ \\
\hline $\mathrm{MoC}$ & $\gamma$-MoC & P-6m2 (187) \\
\hline $\mathrm{Mo}_{2} \mathrm{C}$ & $\beta^{\prime \prime}-\mathrm{Mo}_{2} \mathrm{C}$ & $P-3 m l(164)$ \\
\hline $\mathrm{Mo}_{3} \mathrm{Ga}$ & $\mathrm{Cr}_{3} \mathrm{Si}$ & $P m-3 n(223)$ \\
\hline $\mathrm{Mo}_{6} \mathrm{Ga}_{31}$ & $\mathrm{Mo}_{6} \mathrm{Ga}_{31}$ & $P 12{ }_{l} / c 1$ (14) \\
\hline $\mathrm{Mo}_{8} \mathrm{Ga}_{41}$ & $\mathrm{~V}_{8} \mathrm{Ga}_{41}$ & $R-3 h(148)$ \\
\hline MoGa & $\mathrm{CrGe}$ & $P 2_{1} 3(198)$ \\
\hline $\mathrm{MoGa}_{2}$ & $\mathrm{CrSe}_{2}$ & $P-3 m 1$ (164) \\
\hline$M o G a_{3}$ & $\mathrm{NbGa}_{3}$ & I4/mmm (139) \\
\hline$M o G a_{4}$ & $\mathrm{CrGa}_{4}$ & Im-3m (229) \\
\hline $\mathrm{Mo}_{3} \mathrm{Ga}_{2}$ & $U_{3} S i_{2}$ & P4/mbm (127) \\
\hline $\mathrm{Mo}_{5} \mathrm{Ga}_{3}$ & $\mathrm{Cr}_{5} \mathrm{As}_{3}$ & Pnma (62) \\
\hline $\mathrm{Mo}_{5} \mathrm{Ga}_{4}$ & $\mathrm{Nb}_{5} \mathrm{G} a_{4}$ & $\mathrm{PG}_{3} / \mathrm{mcm}(193)$ \\
\hline $\mathrm{Mo}_{3} \mathrm{C}$ & $\mathrm{Cr} 3 \mathrm{C}$ & Pnma (62) \\
\hline $\mathrm{Mo}_{3} \mathrm{C}_{2}$ & $\mathrm{Cr}_{3} \mathrm{C}_{2}$ & Pnma (62) \\
\hline $\mathrm{Mo}_{2} \mathrm{Ga}_{3}$ & $T i_{2} G a_{3}$ & $P 4 / m(83)$ \\
\hline $\mathrm{Mo}_{2} \mathrm{Ga}$ & $\mathrm{Cu}_{2} \mathrm{Sb}$ & P4/nmm (129) \\
\hline
\end{tabular}

